\title{
Outcomes in Carotid Endarterectomy in a Portuguese tertiary care hospital
}

\section{Correia I. ${ }^{1}$, Meleiro H. ${ }^{1}$, Neves J. ${ }^{2}$, Sousa J. ${ }^{2}$, Afonso G. ${ }^{1}$}

${ }^{1}$ Anaesthesiology Department, ${ }^{2}$ Vascular Surgery Department - Centro Hospitalar São João EPE

\section{Introduction}

\section{Carotid endarterectomy (CEA):}

- Standard treatment of carotid stenosis for symptomatic and asymptomatic patients.

- Indications and outcomes of CEA are based in clinical multicentric trials, where patients and hospitals included were highly selected.

\section{Purpose of the study:}

- Describe patient's characteristics, anaesthesia and surgical techniques, including short-term outcome in our institution.

\section{Materials and methods}

\section{Study:}

- Retrospective

- Patients scheduled for CEA

- Between October 2014-July 2015.

\section{Data obtained from electronic clinical charts:}

- Demographic data

- Anaesthesia technique

- Surgical technique

- Post operative complications (30 days)

- Length of hospital stay

\section{Exclusion criteria:}

- Simultaneous CEA and cardiac surgery

- Absence of data

All analysis were calculated with SPSS 20.0. P-value $<0.05$ was considered to be statistically significant.

\section{Results}

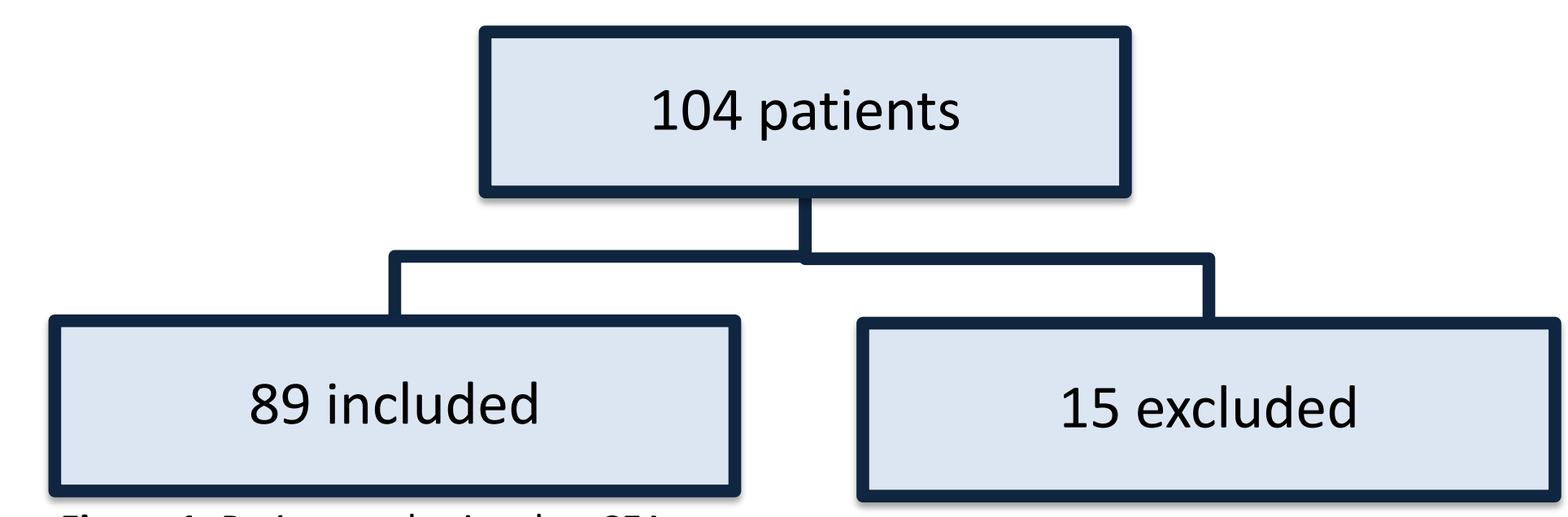

Table 1: Demographic data, anaesthesia and surgical technique, post operative complications (30 days), hospital stay

\begin{tabular}{|c|c|}
\hline \multicolumn{2}{|l|}{ Variables } \\
\hline \multicolumn{2}{|l|}{ Demographic data } \\
\hline Age, median $\left(\mathrm{Q}_{1}, \mathrm{Q}_{3}\right)$ & $62(58-69)$ \\
\hline $\operatorname{Sex}(M)(\%)$ & $83 \%$ \\
\hline Symptomatic patient (\%) & $47 \%$ \\
\hline ASA III (\%) & $100 \%$ \\
\hline \multicolumn{2}{|l|}{ Anaesthesia technique } \\
\hline Cervical Plexus Block (\%) & $93 \%$ \\
\hline General anaesthesia (\%) & $7 \%$ \\
\hline \multicolumn{2}{|l|}{ Surgical technique } \\
\hline Conventional CEA (patch-closure technique) (\%) & $76 \%$ \\
\hline Conventional CEA (direct suture) (\%) & $12 \%$ \\
\hline Eversion CEA (\%) & $12 \%$ \\
\hline Surgery duration median $\left(\mathrm{Q}_{1}, \mathrm{Q}_{3}\right)$ & $118(87-130)$ \\
\hline Cross-clamping time, median $\left(\mathrm{Q}_{1}, \mathrm{Q}_{3}\right)$ & $37(29-50)$ \\
\hline Carotid shunt (\%) & $4 \%$ \\
\hline \multicolumn{2}{|l|}{ Post operative complications (30 days) } \\
\hline Death (\%) & $2 \%$ \\
\hline Stroke (\%) & $4 \%$ \\
\hline Cranial nerve dysfunction (\%) & $4 \%$ \\
\hline Hypertensive crisis (\%) & $43 \%$ \\
\hline Myocardial infarction (\%) & $1 \%$ \\
\hline Pneumonia (\%) & $1 \%$ \\
\hline Cervical hematoma (\%) & $12 \%$ \\
\hline \multicolumn{2}{|l|}{ Length of hospital stay } \\
\hline Intermediate Care Unit, median $\left(\mathrm{Q}_{1}, \mathrm{Q}_{3}\right)$ & $1(1-1)$ \\
\hline Ward, median $\left(\mathrm{Q}_{1}, \mathrm{Q}_{3}\right)$ & $2(1-4)$ \\
\hline
\end{tabular}

\section{Discussion and conclusion:}

A prospective study should determine risk factors of these complications. 